\section{Fairness in der Gesundheitspolitik?}

\author{
T. Schweizer
}

Um es gleich vorwegzunehmen: vor einer Verstaatlichung der Medizin habe ich mich seltsamerweise nie gefürchtet. Ich war schliesslich jahrelang Staatsangestellter in Spitälern und Polikliniken, hatte einen umschriebenen Arbeitsauftrag, einen festen Lohn, eine mit Kollegen und Vorgesetzten geteilte Verantwortung. Die Probleme waren, wenn schon, mehr persönlicher Art. Insgesamt war es fair. Man konnte den Beruf so ausüben. Fairness, so meinte ich seither, wäre bei einer Verstaatlichung der Medizin selbstverständlich. $\mathrm{Zu}$ dieser arglosen Naivität gesellte sich ein Mangel an Vorstellungskraft, wie denn eine solche Verstaatlichung überhaupt ablaufen sollte. Irgendwie stellte ich mir vor, dass man mir und meinen umliegenden Kollegen die Praxis zu einem herabgesetzten Wert abkaufen und uns das zu betreuende Quartier (mit je vergleichbarer Bevölkerungszahl und Altersstruktur) zuteilen würde, unter Bekanntgabe des festen Lohnes. Dies zusammen mit dem Auftrag, Miet- und Lohnverträge mit Angestellten an eine bestimmte Stelle einzuschicken, dazu evtl. die letzten Jahresrechnungen der übrigen Praxisunkosten. In Zukunft würden diese Dinge von dort aus geregelt, ebenso wie die Altersvorsorge und allfällige Krankheitsausfälle. Man würde mir vielleicht auch mitteilen, in welche Spitäler und zu welchen Spezialisten ich entsprechende Patienten zuzuweisen habe. In der Honorarfrage nahm ich an, dass das Prinzip "Gleicher Lohn für gleiche Arbeit» gelte, wobei unter Arbeit Ausbildung, Arbeitsmenge und Einsatzbereitschaft sowie Verantwortung subsumiert wären. Ja, warum denn nicht, wenn die Arbeitsmenge unter den Kollegen einigermassen fair verteilt ist?

Ich meine es durchaus ehrlich. Weniger ehrlich aber ist die Verstaatlichung, wie sie sich jetzt abzeichnet. Unehrlich ist zuerst einmal, dass sie sich nicht als Verstaatlichung zu erkennen gibt, sondern mit den Begriffen "Kostenneutralität» und "Aufhebung des Kontrahierungszwangs" voranschreitet. $\mathrm{Ob}$ es nun Kostenneutralität oder Globalbudget in allen möglichen Bereichen heisst: der durchschnittliche ärztliche Lohn ist damit via Taxpunktbewertung und mit Hilfe des Preisüberwachers staatlich steuerbar. Wir haben dazu kaum etwas zu sagen. Mit der Aufhebung des Kontrahierungszwangs, vordergründig zur Abwehr von «Eurodocs» eingeführt, kann auch die

Korrespondenz:

Dr. med. T. Schweizer

Allg. Medizin FMH

Hessstrasse 47

CH-3097 Liebefeld
Menge der für die soziale Krankenversicherung arbeitenden Ärzte fast beliebig gesteuert werden. Somit könnten die Anzahl ärztlicher Arbeitsplätze und deren Belohnung weitgehend zentral festgelegt werden. Unsere Unkosten, unsere Altersvorsorge und unsere Risiken interessieren den Staat nicht. Es ist unehrlich, dies nicht offen zu sagen, und es ist unfair. Es ist doppelbödig, der Bevölkerung einerseits eine hochstehende und jederzeit verfügbare Gesundheitsversorgung zu versprechen, andererseits diejenigen gering zu achten, die die versprochenen Leistungen zu erbringen versuchen.

Soweit meine naiven und meine bösen Träume: aus letzteren allerdings kann ich nicht mehr so richtig erwachen. Was wäre zu tun? Einfach an das Sprichwort von der heissen Suppe glauben? In kindlicher Weise alle Hoffnungen auf unseren Präsidenten werfen? Oder verdrängen und sich festhalten am Gedanken, man werde es der Ärzteschaft nicht allzu garstig machen können? Nach meiner Meinung ist uns in der Gesundheitspolitik eine etwas verantwortungsvollere Rolle zugedacht. Wir hätten aus unserer Erfahrung heraus Themen zu setzen, statt sie uns immer setzen zu lassen. Hierzu einige Diskussionsvoten:

1. Die Ärzteschaft hat sich die jetzige Entwicklung teilweise selber zuzuschreiben. Sie ist flügellahm, weil sie sich schuldig fühlt für die hohen Gesundheitskosten. Man hat ihr auch lange genug vorgerechnet, wie sie durch ihr Angebot die Nachfrage steigere. Wir müssen uns deshalb nicht wundern, dass die Politiker uns "den Tarif» machen und sogar die Chance wittern, dem komplexen Problem einer qualitativ guten und dennoch bezahlbaren Gesundheitsversorgung mit einer "terrible simplification" zu begegnen. Mehr noch: die Politik erliegt geradezu einer Verdrängung. Verdrängt wird die Tatsache, dass Bemühungen um Krankheit und Gesundheit ein ausserordentlich wichtiger Teil unserer säkularen Kultur sind. Gesundheit ist zum höchsten Gut emporgestiegen und alles, was Krankheit und Leiden bekämpft, hat in unserer verinnerlichten Werteskala höchste Priorität. Entsprechend hoch sind die Anforderungen: der häufigste Vorwurf an Ärzte ist nicht der, zuviel zu tun, sondern den Patienten zu wenig ernst zu nehmen, zu wenig Zeit zu haben, nicht gründlich genug zu untersuchen. Letzteres ist durchaus auch technisch gemeint. Die Hinwendung der Gesellschaft zur Medizin geschieht mit solcher Macht, dass wir auch mit prohibitiven Massnahmen nicht dagegen ankämpfen können. Die tarifarische (Kostenneutralität/Globalbudget) und strukturelle (Aufhebung des Kontrahierungszwangs) Bekämpfung der Ärzte wird möglicherweise bewirken, dass viele von ihnen nicht mehr zum Sozialtarif arbeiten können und als private Anbieter auf dem Markt erscheinen müssen. Die Nachfrage wird nicht abnehmen. Vielleicht kommt diese Entwicklung den Kassen gelegen. Sie könnten danach eine Zusatzversicherung «freie 
Arztwahl" anbieten, in welcher sie von den Regeln, die das BSV in der Grundversicherung setzt, befreit wären. Wird sich der Versicherte am Schluss betrogen fühlen? Wie fair wird er das finden?

2. Die Ärzteschaft zeigt auch in einem anderen Punkt Schwäche: sie lässt sich Schritt für Schritt in einen "double bind" einspannen. Dieser besteht darin, dass uns zu der (meines Erachtens ohnehin $\mathrm{zu}$ grossen) medizinischen Verantwortung nun auch die ökonomische Verantwortung aufgelastet wird. Soweit Managed-Care-Modelle Rationalisierungen in der Begleitung von Kranken bewirken, sind sie zu bejahen. Dort aber, wo sie den Arzt in einen permanenten Konflikt zwischen Erwartungshaltung des Patienten und Sparauftrag der Krankenkasse hineinmanövrieren, sind sie abzulehnen. Warum sagen wir das nicht klarer? Erliegen wir neben Schuldgefühlen auch hartnäckigen Omnipotenzwünschen?

3. Wir brauchen einen Auftrag und einen Lohn. Es gibt einen summarischen Auftrag durch den Staat und einen konkreten Auftrag durch den Kranken. Letzterer ist für unser Handeln entscheidend. Es hat niemand auch nur annähernd den gleichen Einfluss auf ärztliches Handeln wie der Patient. Das müssen sich die Krankenkassen klar vor Augen führen. Unser Ziel bleibt, die Not des Patienten zu lindern, seinen Ängsten zu begegnen und seinen Erwartungen auf sinnvolle Weise zu entsprechen. Der Patient ist letztlich der einzige legitime Auftraggeber, wenn bei seiner Krankheit gespart werden soll. Die Alternative besteht in Rationierungen, welche die Mehrheit des Volkes auf dem Gesetzesweg beschliesst. Versteckte und den (gesetzlich nicht geschützten) Ärzten aufgezwungene Rationierungen sind unfair.

4. Es ist die ganze Gesellschaft, die sich den immer drängender werdenden Fragen nach dem Preis ihres Anspruchs zu stellen hat. Ein für die Antwort notwendiger Such- und Entdeckungsprozess hat wegen der rückwärts gerichteten Sozialpolitik noch gar nicht recht begonnen. In diesem Prozess müsste der einzelne Versicherte Stück für Stück in die Problemlösung einbezogen werden. Das setzt voraus, dass ihm die (entwendete) Verantwortung zurückgegeben wird. Zum Glück sind wir keine Halbgötter in Weiss mehr. Bis zum Patienten, der sich autonom um ärztlichen Rat bemüht, um dann in Eigenverantwortung, auch die Kosten berech- nend, einen Entscheid $\mathrm{zu}$ fällen, ist allerdings noch ein weiter Weg. Es gibt aber keine Alternative zur ökonomischen Partizipation des Versicherten - ausser dem beschriebenen bösen Traum. Krankheit entbindet nicht von Mündigkeit. Natürlich müssen wir Hilflosen und Überforderten beistehen, aber mit dem Ziel der raschestmöglichen Wiederherstellung ihrer Entscheidungsautonomie. Autonomie und Mitverantwortung sind die einzigen tauglichen Heilmittel, einem immer höhere Erwartungen erfüllenden Gesundheitsmarkt Grenzen zu setzen, ohne dessen Qualität zu gefährden. Als konkrete Möglichkeit einer ökonomischen Mitverantwortung sehe ich das individuelle Krankenkassenkonto. Dieses Konto würde bei Krankheit nicht einschmelzen, weil es weiterhin zu einem guten Teil (aber nicht nur!) aus Solidarprämien gespiesen würde. Trotzdem wäre es ein persönliches Konto, das einen individuell wählbaren Versicherungsschutz gewährt und auch einen Spielraum bei der Prämie zulässt, bis hin zur Möglichkeit, in diesem Konto einen Sparanteil anzulegen. Fest definierte Extremkosten wären beim Staat rückversichert. Dem Staat würde somit auch die Rationierungsfrage gestellt.

Letztlich ist es auch ein Akt der Fairness gegenüber der Bevölkerung, wenn sie mehr in die Mitverantwortung und dadurch in die Mitgestaltung unseres Gesundheitswesens einbezogen wird. Wir brauchen ein System, das von allen getragen und bejaht wird, nicht eines, in welchem der Staat immer weniger bezahlt, aber immer mehr befiehlt und damit die Qualität aufs Spiel setzt, ohne im nachhinein dafür die Verantwortung tragen zu müssen.

Der in Rechten und Pflichten emanzipierte Versicherte ist keine Utopie, sondern in guten Teilen schon Realität. Der Patient von heute und/oder sein Umfeld haben einen erstaunlichen Wissensstand in Gesundheitsfragen, sind aber oft ahnungslos hinsichtlich Kostenproblematik. Ersteres macht der in Keimen immer noch patrialistischen Ärzteschaft oft zu schaffen, letzteres schadet uns auf die Länge mehr, als es uns nützt. Fairness im Gesundheitswesen würde bedeuten, dass man uns Ärzten in einem ausgereiften TarMed-Vertrag faire Taxpunktwerte anbietet (siehe oben: Gleicher Lohn für gleiche Arbeit) und dass den Patienten die Chance und der Auftrag gegeben wird, in ihrem Leiden einen einfachen, ehrlichen und kostengünstigen Weg zu finden. Sie und Ihre Berater (Familie/Umfeld/Beratungsstellen/Arzt) werden die von allen beschworene optimale Medizin weit eher bewirken als dies dirigistische Eingriffe tun. 


\section{Facharztprüfung zur Erlangung des Facharzttitels Nephrologie (Innere Medizin)}

Aufgrund des Weiterbildungsprogrammes, welches am 1. Juli 1996 in Kraft gesetzt wurde, ist die Teilnahme an der Facharztprüfung für diejenigen Kandidatinnen und Kandidaten Voraussetzung für die Erlangung des Facharzttitels FMH für Nephrologie, welche ihre Weiterbildung in Nephrologie bis am 30. Juni 1997 nicht abgeschlossen hatten. Es empfiehlt sich, die Facharztprüfung frühestens im letzten Jahr der reglementarischen Weiterbildung abzulegen (Art. 22 WB0).
Ort: Lausanne (im Rahmen der Jahrestagung der Schweizerischen Gesellschaft für Nephrologie)

Datum: 13.-16. Dezember 2000

Prüfungsgebühr: Die SGN erhebt eine Prüfungsgebühr von Fr. 300.-.

Anmeldung:

Prof. F. Brunner, Abteilung für Nephrologie, Kantonsspital, Petersgraben 4, 4031 Basel, Tel. 06126524 10, E-mail: fbrunner@uhbs.ch

Anmeldefrist: 31. August 2000

\section{Schulungs-CD TarMed}

Inhalt:

- Ausgangslage

- Entstehungsgeschichte

- Tarifaufbau (Tarifberechnungen)

- Fallbeispiele/Abrechnungsbeispiele

- Tarifbrowser

FMH-Mitglieder können die CD zum Preis von Fr. 40.- (zuzüglich Fr. 5.50 Porto und Verpackung) beim FMH Tarifdienst bestellen. Aus administrativen Gründen können nur Bestellungen per Fax entgegengenommen werden. Lieferfrist etwa 14 Tage. Die Auslieferung erfolgt per Nachnahme. Für Nicht-Mitglieder beträgt der Preis Fr. 60.- (exkl. Porto und Verpackung).

Hinweis: Die Fallbeispiele basieren noch auf der Version TARMED 2.2. Die Fallbeispiele in der Grundversorgung werden aber durch die Änderung zu TarMed 3 nicht tangiert.

Systemvoraussetzung: Intel Pentium oder vergleichbarer Prozessor, mind. 8 MB RAM, Microsoft Windows 95/98/NT 4.0, Grafikkarte mit einer Auflösung von mindestens $800 \times 600$ und 256 Farben (empfohlen: High-

Color oder TrueColor), CD-ROM Laufwerk; Internet Explorer 4.0.

\section{Bestelltalon}

Name:

Vorname:

Adresse:

Unterschrift:

Anzahl CDs:

FMH Tarifdienst, Fax 0419210586 


\section{Examen de spécialiste en vue de l'obtention du titre de spécialiste FMH en néphrologie (médecine interne)}

Conformément au programme de formation postgraduée qui est entré en vigueur le $1^{\text {er }}$ juillet 1996, la participation à l'examen de spécialiste est une condition requise pour les candidats à l'obtention $\mathrm{du}$ titre de spécialiste FMH en néphrologie qui n'avaient pas terminé leur formation postgraduée en néphrologie le 30 juin 1997. Il leur est recommandé de passer l'examen de spécialiste au plus tôt durant la dernière année de leur formation postgraduée réglementaire (art. 22 RFP).
Lieu: Lausanne (lors de l'assemblée annuelle de la Société suisse de néphrologie)

Date: du 13 au 16 décembre 2000

Taxe d'examen: La SSN prélève une participation aux frais de Fr. 300.--

\section{Inscription:}

Prof. F. Brunner, Division de néphrologie, Hôpital Cantonal, Petersgraben 4, 4031 Bâle, tél. 06126524 10, e-mail: fbrunner@uhbs.ch

Délai d'inscription: le 31 août 2000

\section{CD de formation TarMed}

Contenu:

- Situation initiale

- Historique

- Conception du tarif (calculations)

- Exemples de cas/ exemples de facturation

- Navigateur tarifaire

Les membres de la FMH peuvent commander ce CD-ROM auprès du Service tarifaire de la FMH pour la somme de Fr. 40.- (plus Fr. 5.50 de frais de port et d'emballage). Pour des raisons administratives, seules les commandes par fax peuvent être prises en compte. Le délai de livraison est d'environ 15 jours. La livraison se fait contre remboursement. Pour les non-membres de la FMH, le prix du CD-ROM est de Fr. 60.- (sans les frais de port et d'emballage).

N.B.: les exemples de cas se basent encore sur la version $2.2 \mathrm{du}$ TarMed. Ceux concernant la médecine de premier recours ne sont pas touchés par les modifications de la version $3 \mathrm{du}$ TarMed.

Système informatique nécessaire: Intel Pentium ou processeur similaire, au moins 8 MB de RAM, Microsoft Windows 95/98 NT 4.0, carte graphique avec une résolution d'au moins 800×600 et 256 couleurs (conseil: davantage de couleurs ou couleur vraie), lecteur de CD-ROM; Internet Explorer 4.0.

\section{Bulletin de commande}

Nom:

Prénom:

Adresse:

Signature:

Nombre de CD-ROM désiré:

Service tarifaire FMH, fax 0419210586 